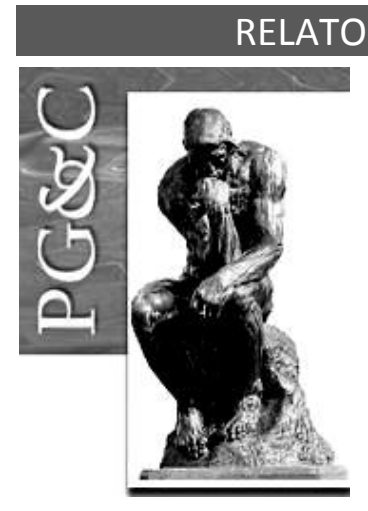

\title{
APLICAÇÃO DO MODELO DE ACEITAÇÃO DE TECNOLOGIA À COMPUTAÇÃO EM NUVEM
}

\author{
Helga Cristina Hedler \\ Doutora em Psicologia Social, do Trabalho e das Organizações pela \\ Universidade de Brasília, Brasil. Professora da Universidade \\ Católica de Brasília, Brasil. \\ E-mail: helgachedler@gmail.com
}

\author{
Edilson Ferneda \\ Doutor em Computação pelo Laboratoire d'Informatique, Robotique et \\ Microélectronique de Monpellier, França. Professor da Universidade \\ Católica de Brasília, Brasil. \\ E-mail: eferneda@pos.ucb.br \\ Bruno Silveira Duarte \\ Mestre em Gestão do Conhecimento e Tecnologia da Informação pela \\ Universidade Católica de Brasília, Brasil. \\ E-mail: seusilveira@gmail.com \\ Hércules Antonio do Prado \\ Doutor em Computação pela Universidade Federal do Rio Grande do Sul, \\ Brasil. Professor da Universidade Católica de Brasília, Brasil. \\ E-mail: hercules@ucb.br
}

\section{Carlos Enrique Carrasco Gutierrez}

Doutor em Economia pela EPGE, Fundação Getúlio Vargas e Doutor em Engenharia Elétrica pela Pontifícia Universidade Católica do Rio de Janeiro, Brasil. Professor da Universidade Católica de Brasília, Brasil.

E-mail: carlosenrique@ucb.br

\begin{abstract}
Resumo
O objetivo dessa pesquisa foi avaliar a aceitação e adoção da computação em nuvem por meio do Modelo de Aceitação de Tecnologia (TAM) e validar um instrumento de obtenção desta medida. Participaram da pesquisa 55 funcionários de uma escola de capacitação para a indústria que utiliza serviços de nuvem (e-mail, armazenamento em disco, agenda, grupos, contatos, bate-papo e web conference). Os resultados indicam que as quatro escalas de medida adotadas (Intenção Comportamental, Utilidade Percebida, Atitude para Uso e Facilidade de Uso Percebida) apresentam confiabilidade boa (Alfa de Cronbach variando entre 0,82 e 0,92). Utilidade Percebida e Atitude para Uso foram preditoras da Intenção Comportamental. Facilidade de Uso Percebida, uma relação não hipotetizada, também foi preditora da Intenção Comportamental. Dois itens da escala Facilidade de Uso Percebida apresentaram cargas fatoriais baixas, o que mostra a necessidade de se reformular ou criar mais itens para avaliar esse construto.
\end{abstract}

Palavras-chave: Computação em nuvem. Modelo de Aceitação de Tecnologia. Comportamento do usuário.

Perspectivas em Gestão \& Conhecimento, João Pessoa, v. 6, n. 2, p. 188-207, jul./dez. 2016. http://periodicos.ufpb.br/ojs2/index.php/pgc. ISSN: 2236-417X. Publicação sob Licença (cc) EY-NC-ND 


\title{
ACCEPTANCE MODEL APPLICATION TECHNOLOGY TO CLOUD COMPUTING
}

\begin{abstract}
The purpose of this study was to evaluate the acceptance and adoption of cloud computing by means of the Technology Acceptance Model (TAM) and to validate an approach to obtain the measures involved. It involved fifty-five employees of a school dedicated to train people from industry that use cloud computing services (e-mail, disk storage, agenda, groups, contacts, chat and web conference). The results pointed out that the four measure scales used (Behavioral Intention, Perceived Usefulness, Attitude Towards Using and Perceived Ease of Use) presented good reliability (Cronbach Alfa varying from 0.82 to 0.92). With respect to the predictive ability, it was observed that Perceived Usefulness and Attitude Towards Using are predictors for Behavioral Intention and Perceived Ease of Use for Behavioral Intention, although the latter relation had not been hypothesized. Two items from Perceived Ease of Use presented low factorial charge, what requires a reformulation or expansion in the items involved in the evaluation for this construct.
\end{abstract}

Keywords: Cloud computing. Technology Acceptance Model. User behavior.

\section{INTRODUÇÃO}

A computação em nuvem refere-se a uma tecnologia baseada na Internet, com a qual informações são armazenadas em servidores remotos, fornecidas como serviço e por demanda de pessoas ou organizações. O impacto das plataformas de computação em nuvem está no fato de oferecerem serviços alternativos ou complementares para as plataformas tradicionais instaladas e mantidas dentro das organizações, e também para os softwares instalados nos computadores pessoais (ZITTRAIN, 2007). Enquanto as plataformas tradicionais foram projetadas para suportar uma escala individual ou organizacional, as plataformas de nuvem podem suportar vários usuários em uma escala tão ampla como a Internet (ETRO, 2009).

Diversos trabalhos destacam as dificuldades técnicas na implementação da computação em nuvem (KHAJEH-HOSSEINI et al., 2011; MARTENS; TEUTEBERG, 2011; YAM et al., 2011; JOHNSON; QU, 2012; KHAJEH-HOSSEINI; SOMMERVILLE; SRIRAM, 2012; AZEEMI; LEWIS; TRYFONAS, 2013; ARMENISE et al., 2014). No entanto, apesar dos benefícios potenciais da adoção da computação em nuvem nas organizações (MANYIKA et al., 2013; CHAVES; SOUZA, 2012), as alterações advindas da adoção de novas tecnologias não se atêm à perspectiva tecnológica. A própria maneira pela qual as empresas gerem seus negócios deve sofrer alterações em todos os niveis organizacionais (RAJ; PERIASAMY, 2011; GONZENBACH; RUSS; BROCKE, 2014).

Vários autores destacam que a aceitação de uma tecnologia está relacionada à qualidade e utilização dos sistemas, à qualidade das informações e à satisfação do usuário (DeLONE; McLEAN, 1992). Segundo Torres (2009), pode haver casos onde uma tecnologia é implantada dentro do prazo, com os valores orçados e os critérios de qualidade e objetivo atingidos, mas que não resulta em benefícios para os clientes ou melhora o desempenho da empresa. Uma das questões que leva ao fracasso parcial ou total deste tipo de processo diz respeito à rejeição dos novos sistemas pelos usuários, ou mesmo à sua utilização parcial ou inadequada (VENKATESH; DAVIS, 2000).

Assim, dada a importância estratégica da informação e das tecnologias da informação e comunicação (TIC) sobre os processos organizacionais (McGEE; PRUZAK, 1997; PORTER, 1986; SANTOS JÚNIOR; FREITAS; LUCIANO, 2005) e considerando o caráter disruptivo da computação em nuvem (MANYIKA et al., 2013) e que toda nova tecnologia pode enfrentar resistência na sua adoção, é pertinente avaliar os fatores associados à sua aceitação e uso em um ambiente organizacional.

Perspectivas em Gestão \& Conhecimento, João Pessoa, v. 6, n. 2, p. 188-207, jul./dez. 2016. 
Vários modelos para mensurar a intenção de uso e aceitação de tecnologias foram propostos e testados empiricamente nos últimos anos para compreender sua adoção pelo usuário, tais como a Teoria da Ação Racional (TRA) de Fishbein e Ajzen (1975), o Modelo de Aceitação Tecnológica (TAM), desenvolvido por Davis (1986) e revisado por Davis, Bagozzi e Warshaw (1989), a Teoria do Comportamento Planejado (TPB), de Ajzen (1991), a Teoria da Difusão de Inovações, de Rogers (1995) e o Modelo de Sucesso, de DeLone e McLean (1992). Esses modelos foram aplicados a uma variedade de tecnologias de informação em diferentes contextos e populações.

Essa pesquisa teve como objetivo validar um instrumento baseado no TAM para o contexto da computação em nuvem. Os objetivos específicos incluíram a análise da confiabilidade das escalas de Utilidade Percebida, Facilidade de Uso Percebida, Atitude para Uso, Intenção Comportamental e Uso Real.

\section{REFERENCIAL TEÓRICO}

TAM foi utilizado nesta pesquisa devido ao aporte teórico e empírico que vem recebendo em suas aplicações (SAGA; ZMUD, 1993). Partiu-se do pressuposto de que utilizar e adaptar uma medida com base neste modelo para identificar aspectos da aceitação de uma tecnologia ajuda na prevenção de situações que levam ao fracasso ou ao uso efetivo dessa tecnologia após sua adoção (DAVIS, 1989).

A computação em nuvem é uma tecnologia recente e que vem sendo adotada por organizações de todos os portes (MORRIS, 2014). Soluções de nuvem são normalmente baseadas em um modelo de autosserviço. Isto altera significativamente as tarefas e serviços prestados pelos setores responsáveis pela área de TI e, embora de forma não tão acentuada, também exige novas habilidades para o trabalhador da área de negócio em relação à sua interação com a TI. Há de se pensar computação em nuvem como uma maneira de organizar o potencial da Internet para o provimento de serviços distribuídos, trazendo novos produtos e recursos para o trabalho.

A computação em nuvem traz uma mudança de paradigma e um novo conjunto de objetos técnicos que os usuários terão de lidar para a realização de seus trabalhos. Daí a necessidade de se entender a aceitação dessa tecnologia no contexto das organizações (PIRES, 2007; TAURION, 2009). Para esclarecer as mudanças mais perceptíveis no mundo do trabalho com relação ao usuário diante da adoção de computação em nuvem, apresenta-se no Quadro 1 um comparativo das maneiras de realização de um trabalho com e sem a adoção da computação em nuvem.

Quadro 1 - Trabalho com e sem computação em nuvem

\begin{tabular}{|l|l|}
\hline \multicolumn{1}{|c|}{ Trabalho sem computação em nuvem } & \multicolumn{1}{c|}{ Trabalho com computação em nuvem } \\
\hline $\begin{array}{l}\text { Utiliza-se o computador no local de trabalho } \\
\text { para acessar o servidor da organização }\end{array}$ & $\begin{array}{l}\text { Utilizam-se quaisquer tipos de dispositivos digitais } \\
\text { conectados à Internet para acessar a nuvem de } \\
\text { informações da organização em qualquer } \\
\text { momento e em qualquer lugar. }\end{array}$ \\
\hline $\begin{array}{l}\text { Todos os softwares e serviços da organização } \\
\text { estão instalados no dispositivo do trabalhador }\end{array}$ & $\begin{array}{l}\text { O trabalhador não precisa ter os softwares } \\
\text { instalados. Realiza o acesso a partir de um } \\
\text { navegador de Internet. }\end{array}$ \\
\hline $\begin{array}{l}\text { Recorre ao suporte interno de TI sempre que há } \\
\text { um problema de TI }\end{array}$ & $\begin{array}{l}\text { A própria nuvem resolve o problema por meio da } \\
\text { colaboração e da larga extensão do uso das } \\
\text { ferramentas, que acaba por minimizar incidências } \\
\text { de erros e problemas. }\end{array}$ \\
\hline $\begin{array}{l}\text { Tem limite de armazenamento e } \\
\text { processamento }\end{array}$ & $\begin{array}{l}\text { Entra num processo de escalonamento de } \\
\text { demanda quanto ao armazenamento e }\end{array}$ \\
\hline
\end{tabular}

Perspectivas em Gestão \& Conhecimento, João Pessoa, v. 6, n. 2, p. 188-207, jul./dez. 2016. 
processamento.

Fonte: Autoria própria

Na perspectiva de Hatch (1993), a introdução de uma nova tecnologia, em um primeiro momento, representaria a materialização de expectativas e valores culturais de determinados grupos, como o gerencial. Em um segundo momento, seria percebida como um novo artefato por grupos operacionais que não participaram de sua definição e desenho. Os grupos operacionais poderiam, então, aceitá-la, rejeitá-la ou ignorá-la. De qualquer maneira, a mesma tecnologia passa a ser alvo de um processo de redefinição e ajuste de valores e expectativas da cultura organizacional.

Segundo Betiol e Tonelli (2001), os funcionários não se veem como determinantes para o sucesso ou o fracasso da implantação de novas plataformas de TI. Todavia, uma ação gerencial de planejamento, quando conduzida de maneira participativa, pode ser um fator determinante para implantação, aceitação e sucesso de novas tecnologias na organização. Estudos em planejamento de gestão da TI (PIRES, 2007; SHAPIRO; VARIAN, 1999) mostram que, nesse contexto, ações desse tipo podem reduzir obstáculos, como a resistência dos gestores.

Porras e Robertson (1992, p. 724) argumentam que "mudança no comportamento dos membros da organização é a essência da mudança organizacional". Qualquer mudança bem sucedida irá persistir por longo tempo somente se, em resposta às mudanças nas características organizacionais, os membros alterarem seu comportamento e o trabalho de maneira apropriada. Assim, no campo da adoção de $\mathrm{Tl}$, evidencia-se a necessidade de utilização de um modelo que investigue a percepção, a intenção comportamental e o uso real de sistemas para produzir evidências preparativas para a mudança de TI na organização.

Provavelmente, a adoção ou resistência à tecnologia será resultado de um conjunto de variáveis, dentre as quais as crenças e manifestações coletivas que advêm das abstrações e comportamentos dos indivíduos, além do planejamento e do contexto organizacional no qual se insere e se incorpora (ou não) uma nova tecnologia. Ao partir desta compreensão, escolheuse TAM para auxiliar a diagnosticar a aceitação e uso da nova tecnologia como um fenômeno inerente à mudança.

\subsection{Modelo de Aceitação de Tecnologia}

TAM é uma adaptação do modelo da Teoria da Ação Racional (TRA) (DAVIS, 1986). Na TRA, a intenção determina o comportamento real, que se refere aos atos passíveis de observação (FISHBEIN; AJZEN, 1975). A aplicação da TRA possibilita prever a escolha das pessoas em situações diversas, a partir da definição de relações entre crenças, atitudes, normas, intenções de comportamento e o próprio comportamento. Ainda, as crenças e a norma subjetiva influenciam a atitude para a ação ou comportamento. Por sua vez, atitude para ação ou comportamento e norma subjetiva determinam a intenção comportamental que leva ao comportamento em si, ou seja, a ação do individuo.

De acordo com sua crença, uma pessoa toma uma atitude favorável ou desfavorável em relação a algo, a outra pessoa ou a determinada tecnologia (FISHBEIN; AJZEN, 1975). Assim como a atitude direciona a ação, ela também possui um componente avaliativo que influencia a intenção comportamental. Esta, por sua vez, é também influenciada pela norma subjetiva compartilhada pela maioria das pessoas de um determinado grupo. Desta forma, ambas, norma subjetiva e atitude, são variáveis preditoras de intenção comportamental. A consequência do modelo é o comportamento, ou seja, a ação (DAVIS, 1986).

No entanto, Davis, Bagozzi e Warshaw (1989) não incluíram no TAM a norma subjetiva como determinante da intenção comportamental, pois: 
[...] É difícil separar os efeitos diretos da norma subjetiva em intenção comportamental. Normas subjetivas podem influenciar indiretamente a intenção comportamental, mas ao contrário de se imaginar que geralmente o uso do computador por gestores e profissionais é voluntário, em alguns casos, as pessoas podem utilizar um sistema de forma simplesmente a cumprir com ordens de superiores, e não devido a seus próprios sentimentos e crenças sobre seu uso, que poderiam advir de uma norma subjetiva.

Davis (1986) argumenta que usuários formam tendências motivacionais logo depois de serem expostos a um novo sistema ou projetos e suas respectivas potencialidades. Seria também possível verificar consequências comportamentais como tendências de aceitação ou não. O processo de teste da aceitação consiste em breve demonstração de novos sistemas, utilizando o próprio sistema ou recursos multimídia de apresentação, seguido da aplicação de uma medida para verificar a motivação para o uso do sistema no trabalho (DAVIS, 1986). Essa é a visão geral do modelo para descrever o processo motivacional entre a tecnologia e o comportamento do usuário. Uma versão mais recente do TAM foi apresentada por Davis, Bagozzi e Warshaw (1989). Sua estrutura é apresentada na Figura 1.

Figura 1 - Modelo de Aceitação de Tecnologia

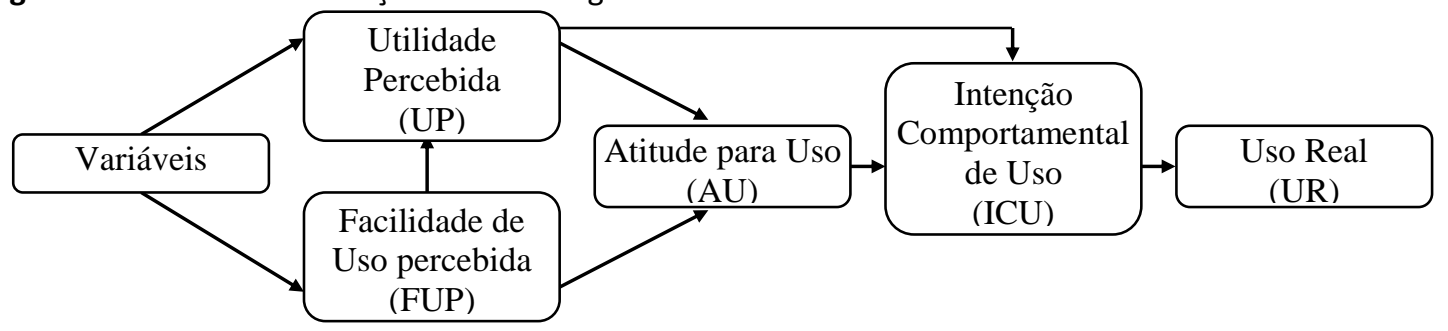

Fonte: Davis, Bagozzi e Warshaw (1989)

No TAM, variáveis externas referem-se às características de sistemas, como menus, ícones e interface, ou seja, a tecnologia em si, sob a qual se está investigando a aceitação. Utilidade percebida é compreendida como a possibilidade de conceber vantagem ao desempenho em determinada atividade. Percepção de facilidade de uso refere-se à ideia da necessidade de pouco ou nenhum esforço para utilizar determinado sistema (DAVIS, 1989). Atitude para uso é o "[...] grau individual de avaliação que vai influenciar a intenção de comportamento" (FISHBEIN; AJZEN, 1975, p. 216). Intenção comportamental é "[...] uma probabilidade subjetiva individual que determina o comportamento" (FISHBEIN; AJZEN, 1975, p. 288). Portanto, o uso real é uma função de avaliação da frequência de uso, no caso de sistemas já disponibilizados, ou intenção de uso futuro, no caso do desenho de novos sistemas (DAVIS, 1989, p. 989).

Davis, Bagozzi e Warshaw (1989) aplicaram o modelo em dois estudos, o primeiro desenvolvido a partir de um sistema em uso e o segundo sobre um sistema que ainda viria a ser utilizado. As medidas de utilidade percebida e facilidade de uso percebida foram refinadas e simplificadas, resultando em dois blocos de seis questões cada, com margem de confiabilidade para utilidade percebida de 0,98 e para facilidade de uso percebida de 0,94 (DAVIS; BAGOZZI; WARSHAW, 1989).

Todavia, a gênese do modelo (DAVIS; BAGOZZI; WARSHAW, 1989) previa que ele oferecesse uma estrutura teórica essencial sobre os determinantes da aceitação (utilidade percebida e da facilidade de uso percebida) e que tornasse possível a inserção de outras variáveis. Neste sentido, Venkatesh e Davis (2000) propuseram uma nova representação do 
modelo, o TAM2, que mantinha a essência do primeiro, mas sugeria algumas variáveis externas (experiência, voluntariado, norma subjetiva, imagem, relevância para o trabalho, qualidade da entrega, demonstração do resultado), anteriores às variáveis do corpo do modelo de Davis, Bagozzi e Warshaw (1989).

O modelo de aceitação de tecnologia tem sido usado e adaptado em muitas pesquisas. Por exemplo, Alharbi (2012) desenvolveu e aplicou um modelo da aceitação de computação em nuvem junto a funcionários de empresas de tecnologia da informação na Arábia Saudita. Sek et al (2010) utilizaram o modelo de Davis (1989) com seus cinco construtos (Utilidade Percebida - UP; Facilidade de Uso Percebida - FUP; Atitude para Uso - AU; Intenção Comportamental de Uso - IC e Uso Real - UR) como linha de base para verificar as relações entre as variáveis do modelo, assim como testá-las no contexto de uso da tecnologia smartphone por usuários de alto nível de escolaridade.

Sek et al (2010) utilizaram uma amostra de conveniência: 60 participantes que se inscreveram no curso de Sistemas Digitais, e eram potenciais usuários de smartphones. Os inscritos que possuíam experiência prévia com utilização de smartphones foram eliminados da análise, resultando em uma amostra final de 40 usuários. Antes de realizar a coleta de dados final, os autores conduziram um teste para determinar a confiabilidade e a validade discriminante das escalas de medição propostas no modelo TAM. A análise da confiabilidade do modelo TAM usado para avaliar a aceitação e uso do smartphone foi determinada por meio da consistência interna dos itens e do alfa de Cronbach (TABACHNICK; FIDELL, 2001). Os construtos originais do TAM (FUP; UP; AU; IC e UR) obtiveram coeficientes de confiabilidade variando entre 0,91 e 0,98 .

Segundo Venkatesh et al (2003, p. 428), a evolução do TAM demonstra que o modelo pode ser usado para contextos de sistemas de informação para prever aceitação e uso de tecnologia no ambiente de trabalho. Chuttur (2009) resumiu sua evolução, suas principais aplicações, extensões, limitações e críticas, concluindo que "[...] embora o TAM seja um modelo altamente citado, pesquisadores possuem divergências e convergências em relação aos seus pressupostos teóricos e sua eficácia prática" (CHUTTUR, 2009, p. 10).

Apesar de contribuições significativas ao modelo TAM, as diferentes aplicações independentes realizadas a fim de adaptá-lo aos ambientes de constante mudança de TI e sua expansão, levou a um estado de caos e confusão teórica em que constantemente são inseridos apêndices ao modelo original e que trazem uma ilusão com relação ao acúmulo de conhecimento sobre o modelo. Ou seja, o TAM cumpre o seu propósito, mas é tempo de se propor novos modelos (BENBASAT; BARKI, 2007; CHUTTUR, 2009).

Uma limitação do modelo TAM, segundo Benbasat e Barki (2007) e Chuttur (2009), seria o seu médio alcance, que fornece uma ponte potencialmente útil sobre antecedentes e consequentes da aceitação, mas que se limita a isto. Como um exemplo de modelo expandido do TAM, Benbasat e Barki (2007) sinalizam a pesquisa de Wixom e Todd (2005), que propuseram informações e perspectivas do sistema de qualidade, abrindo o caminho para o próximo passo no desafio de identificar os artefatos de TI e as características que influenciam sua adoção, para assim melhorar as variáveis de estudo da aceitação.

Expandir o modelo foge do propósito desta pesquisa. Apesar de o TAM ter sido amplamente aplicado em outros países, no Brasil seu uso é incipiente, e a confusão teórica também é perceptível, de modo que esta pesquisa poderá abrir o campo para que outros estudos possam abarcar novas variáveis como fizeram Wixom e Todd (2005). Adaptar o TAM para utilizá-lo na análise da aceitação de computação em nuvem, no contexto organizacional brasileiro, foi o foco desta pesquisa.

Devido à sua fidelidade ao modelo TAM e à relevância dos resultados alcançados por Sek et al (2010), optou-se por utilizar seu instrumento como base para a adaptação e realização desta pesquisa sobre aceitação de computação em nuvem. 


\subsection{Computação em nuvem}

A computação em nuvem tem uma série de definições. Do ponto de vista técnico, o National Institute of Standards and Technology (NIST), do Ministério do Comércio americano, define a computação em nuvem como:

[...] um modelo para habilitar o acesso por rede ubíquo, conveniente e sob demanda a um conjunto compartilhado de recursos de computação (como redes, servidores, armazenamento, aplicações e serviços) que possam ser rapidamente provisionados e liberados com o mínimo de esforço de gerenciamento ou interação com o provedor de serviços. (MELL; GRANCE, 2011, p. 2).

Na perspectiva de negócios, a computação em nuvem é definida como:

[...] um modelo de serviço de tecnologia da informação onde os serviços de computação (hardware e software) são entregues sob demanda para os clientes em uma rede na forma de autoatendimento, independente do dispositivo e localização. Os recursos necessários para fornecer o requisito Níveis de Qualidade de Serviço são compartilhados, dinamicamente escaláveis e rapidamente provisionados, virtualizados e liberados com interação mínima com o provedor de serviço. Os usuários pagam pelo serviço como despesa operacional, sem incorrer em despesas de capital inicial significativo, com os serviços de nuvem empregando um sistema de medição que divide o recurso de computação em blocos apropriados. (NIST, 2009 apud MARSTON et al., 2011, p. 177)

A computação em nuvem tem sido vista como um passo na mercantilização dos investimentos em TI (CARR, 2003), ou ainda como o resultado de uma evolução para um modelo de negócio utilitário. Nesse contexto, recursos de computação são fornecidos como um serviço (RAPPA, 2004), e também como elemento central da era da Web 2.0, em que a Internet é usada como uma plataforma de software (O'REILLY, 2012), ou simplesmente como uma aplicação do poder de escala e de generalização da Internet (ZITTRAIN, 2007). O termo é utilizado também para se referir a um sistema baseado em servidores virtuais e/ou físicos disponibilizados em nuvem.

Segundo Velte, Velte e Elsonpeter (2012, p. 32), computação em nuvem “é uma ideia que permite utilizar as mais variadas aplicações via Internet, em qualquer lugar e independente da plataforma, com a mesma facilidade de tê-las instaladas no computador". Uma nuvem é utilizada, nos diagramas de rede, para retratar a Internet. Para estes autores, em um sentido topológico, uma solução de computação em nuvem muitas vezes é compreendida como uma metáfora da própria Internet, e esse conceito agrega clientes, data center e servidores interligados como no esquema da Figura 2. 
Figura 2 - Topologia básica da computação em nuvem

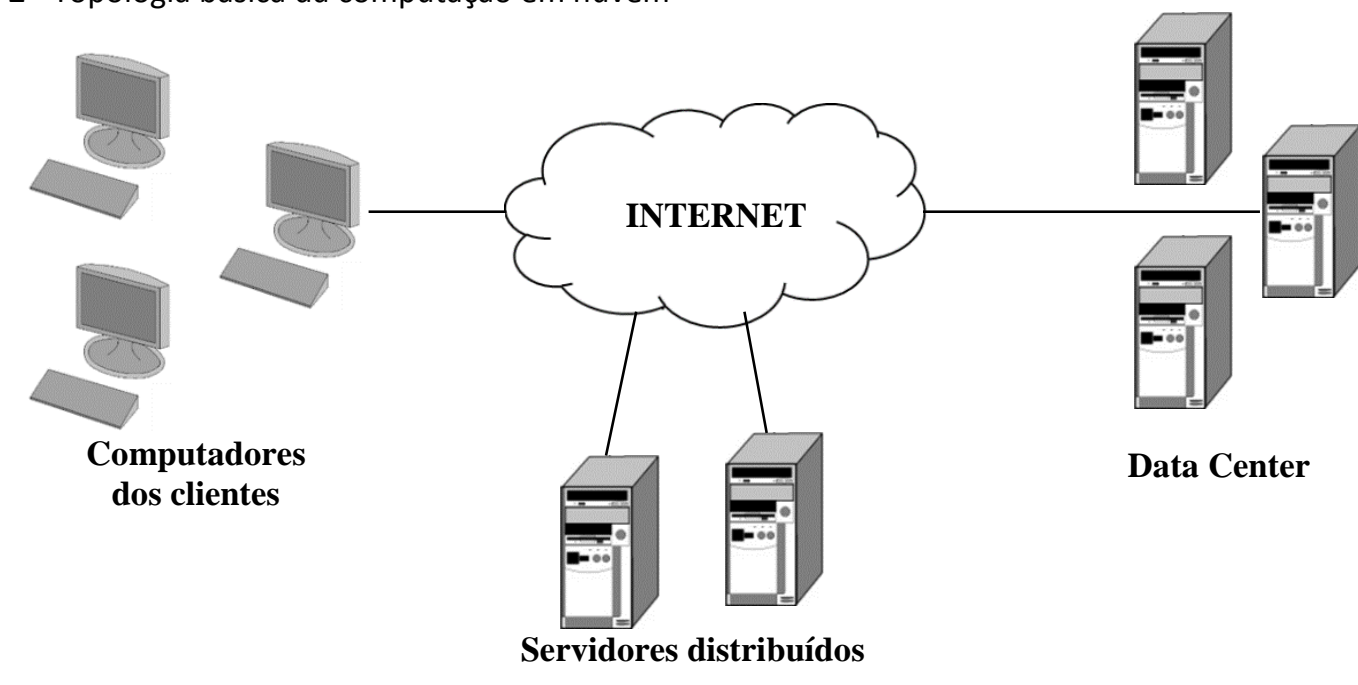

Fonte: Velte, Velte e Elsenpeter (2012)

Os clientes são as pessoas que utilizam dispositivos para gerenciar sua informação na nuvem. Os dispositivos dos clientes geralmente se enquadram em uma das seguintes três categorias: dispositivos móveis, que incluem smartphones; clientes thin - computadores que não têm disco rígido interno, mas exibem as informações; e os clientes thick, que utilizam um navegador instalado em um computador pessoal para acessar a nuvem (VELTE; VELTE; ELSENPETER, 2012).

Data center é um conjunto de servidores onde o software e os dados são armazenados. Pode estar localizado fisicamente em qualquer lugar do mundo e ser acessado via Internet. Uma tendência mundial da TI é a virtualização de servidores. Isto é, o software pode ser instalado, permitindo a utilização de vários servidores virtuais funcionando em um único servidor físico. Entretanto, os servidores não precisam estar alocados em um mesmo local físico. Isto permite ao prestador de serviços maior flexibilidade nas opções e na segurança. De acordo com Velte, Velte e Elsenpeter (2012, p. 34), "a estrutura pode ser implantada de várias maneiras diferentes e isto dependerá de como o provedor escolheu construir a solução da nuvem".

As representações da arquitetura de computação em nuvem dependem de como a organização opta por organizar sua nuvem. Hoje, as pessoas e as organizações dispõem de vários tipos de dispositivos para conexão com a nuvem e é comum que estes sejam utilizados nas configurações da solução. De forma similar, um número significativo de aplicativos está sendo disponibilizado para ser instalado nos dispositivos móveis e acessar a nuvem para executar parte dos serviços ou tarefas para quais foram criados. Também é comum as pessoas adquirirem dispositivos móveis (smartphones, por exemplo) com um sistema operacional ou plataforma embarcados e que recorrem à Internet para a realização de parte dos serviços. Porém, uma vez que a computação em nuvem é ainda um paradigma em evolução, diferentes representações vêm sendo propostas. Nesse sentido, Buyya et al (2012) preveem que as definições, casos de uso, tecnologias, problemas, riscos e benefícios da computação em nuvem serão redefinidos em debates entre os setores público e privado e essas definições, atributos e características evoluirão com o tempo. Muitas organizações de $\mathrm{TI}$, como Google, Oracle, Amazon, Salesforce, IBM, Microsoft e Sun Microsystems, estão investindo recursos consideráveis na ampliação de centros de processamento de dados para a camada de infraestrutura da nuvem, em diversos locais do mundo, bem como estão desenvolvendo seus 
produtos de software e plataformas.

Para Armbrust et al (2009, p. 5), "computação em nuvem é um termo novo para um sonho de longa data da computação como uma utilidade, e que surgiu recentemente como uma realidade comercial". O autor apresenta como desafios e oportunidades para o crescimento da computação em nuvem os itens elencados no Quadro 2.

Quadro 2 - Desafios e oportunidades para o crescimento da computação em nuvem

\begin{tabular}{|c|c|c|}
\hline$\#$ & Desafios & Oportunidade de pesquisa \\
\hline 1 & Disponibilidade de serviço & $\begin{array}{l}\text { - Estudar o uso múltiplo e simultâneo de provedores em nuvem } \\
\text { - Desenvolver elasticidade para prevenir quedas }\end{array}$ \\
\hline 2 & Bloqueio de dados & $\begin{array}{l}\text { - Padronizar APIs (Application Programming Interface, ou } \\
\text { Interface de Programação de Aplicativos) }\end{array}$ \\
\hline 3 & $\begin{array}{l}\text { Confidencialidade e auditoria } \\
\text { de dados }\end{array}$ & $\begin{array}{l}\text { - Implantar criptografia e firewalls (dispositivo responsável pela } \\
\text { política de segurança de uma rede de computadores) } \\
\text { - Estudar a questão da confidencialidade de dados } \\
\text { armazenados em países diferentes }\end{array}$ \\
\hline 4 & $\begin{array}{l}\text { Gargalos na transferência de } \\
\text { dados }\end{array}$ & $\begin{array}{l}\text { - Aprimorar backup/arquivamento de dados } \\
\text { - Aumentar a taxa de transferência nos switches }\end{array}$ \\
\hline 5 & $\begin{array}{l}\text { Imprevisibilidade de } \\
\text { desempenho }\end{array}$ & $\begin{array}{l}\text { - Melhorar o suporte de máquinas virtuais } \\
\text { - Aumentar memória flash (chip que preserva conteúdos } \\
\text { regraváveis sem a necessidade de fonte de alimentação) } \\
\text { - Gerir equipes de contenção }\end{array}$ \\
\hline 6 & Armazenamento escalável & $\begin{array}{l}\text { - Desenvolver sistemas de previsão e provisionamento } \\
\text { adequado de hardware/storage (unidades voltadas para o } \\
\text { armazenamento seguro de grandes volumes de dados) }\end{array}$ \\
\hline 7 & $\begin{array}{l}\text { Erros em grandes sistemas } \\
\text { distribuídos }\end{array}$ & - Criar um fragmentador independente \\
\hline 8 & $\begin{array}{l}\text { Escalar rapidamente a } \\
\text { capacidade segundo } \\
\text { demanda }\end{array}$ & - Desenvolver ferramentas automáticas de escalonamento \\
\hline 9 & Licenciamento de software & - Aprimorar sistemas de licenciamento \\
\hline 10 & Comportamento do usuário & $\begin{array}{l}\text { - Pesquisar níveis de aceitação do usuário com relação à } \\
\text { adoção }\end{array}$ \\
\hline
\end{tabular}

Fonte: Adaptado de Armbrust et al (2009)

Como uma das questões que direciona ao fracasso parcial ou total do processo de adoção de tecnologias se refere à não aceitação dos novos sistemas pelos usuários ou mesmo a sua utilização parcial ou inadequada (VENKATESH; DAVIS, 2000), soma-se ao quadro de Armbrust et al (2009) o desafio de abordar a perspectiva comportamental do usuário para o estudo da aceitação de computação em nuvem. Nesse sentido, Alharbi (2012) destaca que há ainda poucos estudos que enfocam a perspectiva comportamental, além da escassez de pesquisas sobre mudanças e impactos para as pessoas no mundo do trabalho, provenientes da entrada e expansão do uso de computação em nuvem. Isso a despeito de existirem publicações sobre os possíveis impactos da computação em nuvem no trabalho (MILLER, 2009; TAURION, 2009).

\section{ASPECTOS METODOLÓGICOS}

Nesta pesquisa, foi utilizado o modelo TAM, com base no instrumento de pesquisa de Sek et al (2010), mas não foram testadas as hipóteses preconizadas no modelo, haja vista que o objetivo desta pesquisa é testar a escala de medida junto a usuários da computação em 
nuvem. O modelo utilizado é formado pelas variáveis UP, FUP, AU, IC e UR, que formam a estrutura básica do TAM, de acordo com o esquema apresentado na Figura 3.

Figura 3 - Modelo TAM adotado

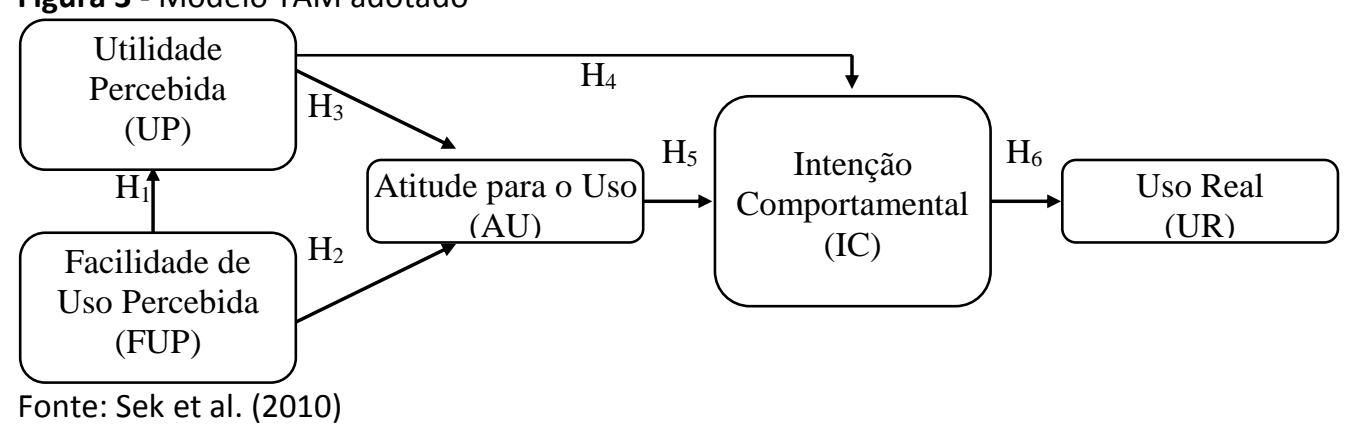

As variáveis consideradas são assim definidas:

- Utilidade Percebida (UP) está associada ao "grau que cada pessoa acredita que o uso de um sistema em particular poderia aumentar seu desempenho de trabalho" (DAVIS, 1986, p. 12);

- Facilidade de Uso Percebida (FUP) "refere-se ao grau em que uma pessoa acredita que o uso de um sistema em particular estaria livre de esforço" (DAVIS, 1986, p. 13);

- Atitude para Uso (AU) é o "grau individual de avaliação que vai influenciar a intenção de comportamento" (FISHBEIN; AJZEN, 1975, p. 216);

-Intenção Comportamental (IC) refere-se a "uma probabilidade subjetiva individual que determina o comportamento" (FISHBEIN; AJZEN, 1975, p. 288);

- Uso Real (UR) foi definido como uma função de avaliação da frequência de uso da computação em nuvem.

\subsection{Instrumento de coleta de dados}

Os dados da pesquisa foram coletados via questionário disponibilizado na Internet. A revisão de literatura sobre o TAM permitiu identificar medidas existentes para os construtos que pudessem avaliar a aceitação e uso da computação em nuvem. As escalas de cada uma das variáveis consideradas foram adaptadas do estudo de Davis, Bagozzi e Warshaw (1989) e Sek et al (2010). Os itens foram reescritos para o contexto do estudo. Uma escala Likert de 5 pontos de discordo fortemente a concordo fortemente foi usada para mensurar os itens. 0 instrumento foi dividido em duas sessões no questionário, a primeira com 21 itens usados para mensurar todas as variáveis independentes que afetam a aceitação e uso da computação em nuvem. A segunda sessão contém questões relativas aos dados sociodemográficos dos participantes. Os elementos que compuseram o questionário são apresentados no Quadro 3. 
Quadro 3 - Instrumento de pesquisa

Facilidade de uso percebida (FUP)

FUP $_{1}$ Aprender a usar ferramentas de computação em nuvem seria fácil para mim.

$\mathrm{FUP}_{2}$ Eu sei facilmente como proceder com as ferramentas de computação em nuvem para fazer o que eu quero.

$\mathrm{FUP}_{3} \mathrm{~A}$ maneira de interação com as ferramentas de computação em nuvem é clara e facilmente compreendida.

$\mathrm{FUP}_{4}$ As ferramentas de computação em nuvem são flexíveis para que eu possa usá-las da maneira que melhor me convier.

FUP $P_{5}$ Seria fácil ficar mais habilidoso no uso das ferramentas de computação em nuvem.

FUP $_{6}$ Eu considero ferramentas de computação em nuvem fáceis de usar.

\section{Utilidade percebida (UP)}

UP $_{1}$ Usando ferramentas de computação em nuvem meu trabalho fica mais rápido.

$U_{2}$ Usando ferramentas de computação em nuvem meu desempenho melhora.

$U_{3}$ Usando ferramentas de computação em nuvem minha produtividade aumenta.

$\mathrm{UP}_{4}$ Meu trabalho fica mais efetivo (eficiente e eficaz) usando ferramentas de computação em nuvem.

$U_{5}$ Meu trabalho fica mais fácil usando ferramentas de computação em nuvem.

UP $_{6}$ Ferramentas de computação em nuvem são úteis para o meu trabalho.

\section{Atitude para uso (AU)}

$\mathrm{AU}_{1} \quad$ Utilizar ferramentas de computação em nuvem é uma ótima ideia.

$\mathrm{AU}_{2}$ Eu desejo utilizar ferramentas de computação em nuvem.

$\mathrm{AU}_{3}$ Seria muito melhor utilizar ferramentas de computação em nuvem.

$\mathrm{AU}_{4}$ Eu gosto da ideia de utilizar ferramentas de computação em nuvem no trabalho.

Intenção Comportamental (IC)

IC $_{1}$ Eu pretendo utilizar ferramentas de computação em nuvem, sempre que possível.

$I_{2}$ Eu tenho a intenção de aumentar o uso de ferramentas de computação em nuvem.

$\mathrm{IC}_{3}$ Eu adotaria novas ferramentas de computação em nuvem, no futuro.

Uso real (UR)

UR $_{1}$ Quais ferramentas de computação em nuvem você utiliza?
( ) E-mail
( ) Armazenamento (disco)
() Agenda
( ) Contatos
( ) Webconferência (hangout)
( ) Bate-papo
( ) Grupos

$\mathrm{UR}_{2}$ Com que frequência você usa ferramentas de computação em nuvem?
( ) Todos os dias
() Duas vezes por semana
( ) Três vezes por semana
() Quatro vezes por semana () Cinco ou seis vezes por semana

\section{Dados Sociodemográficos (DS)}

DS 1 Sexo:

() Masculino ( ) Feminino

$\mathrm{DS}_{2}$ Maior Formação Concluída:
( ) Pós-Doutorado
() Doutorado
() Mestrado
( ) Especialização
( ) Graduação
( ) Técnico
( ) Fundamental

$\mathrm{DS}_{3}$ Área de Formação:
( ) Ciências Exatas e da Terra
( ) Ciências Biológicas
( ) Engenharias
( ) Ciências da Saúde
( ) Ciências Sociais Aplicadas
( ) Ciências Humanas
( ) Linguística, Letras e Artes
( ) Multidisciplinar

$\mathrm{DS}_{4}$ Área de Atuação:
( ) Educação Profissional e Tecnológica
( ) Tecnologia e Inovação
( ) Relações com o Mercado
() Relações Internacionais
( ) Administração
() Prospectiva Profissional
( ) Orçamento e Financeiro
() Recursos Humanos
( ) Tecnologia da Informação

DS5 Faixa Etária:
( ) $\leq 21$ anos
( ) $>21$ e $\leq 30$ anos
( ) $>30$ e $\leq 39$ anos
() $>39$ e $\leq 49$ anos
() $>49$ e $\leq 59$ anos
() $\geq 60$ anos

Fonte: Adaptado de Sek et al. (2010) 


\subsection{Tecnologia avaliada}

A pesquisa foi realizada em uma escola de capacitação para a indústria, aonde o uso da computação em nuvem teve início em meados de 2012 para serviços de comunicação digital e armazenamento de informações providos pela Google (e-mail, armazenamento em disco, agenda, grupos, contatos, bate-papo e web conference).

\subsection{Participantes}

O universo da pesquisa foi os 78 funcionários que atuam em duas unidades fim do negócio da organização, para identificar a perspectiva da aceitação do usuário final da tecnologia. A participação na pesquisa foi livre e voluntária. O questionário foi enviado a todos os funcionários das Unidades, sendo que a amostra final contou com 54 respondentes. Pasquali (2012, p. 145) pondera que o tamanho da amostra para a realização da analise fatorial não é consensual, e enfatiza:

[...] que o tamanho desejado de uma amostra depende do tamanho das cargas fatoriais obtidas. Com cargas fatoriais em torno de 0,80 , obtêm-se soluções fatoriais altamente estáveis em amostras de 50 pessoas. Quando as cargas fatoriais estão ao redor de 0,40 , porém, amostras de 300 a 400 sujeitos são necessárias para atingir soluções estáveis.

Dentre os participantes, 30 (56\%) são do sexo masculino e 24 (44\%) do sexo feminino. Predominou a faixa etária de 40 a 49 anos (23, ou 42,6\%), seguida da faixa de 31 e 39 anos (11, ou $20 \%$ ), de 22 a 30 anos (10, ou 18,5\%), de 18 a 21 anos (4, ou 7,4\%), de 50 a 59 anos (4, ou $7,4 \%$ ) e 60 anos ou mais (2, ou 3,7\%). Com relação ao nível de formação, 5,6\% (3) são técnicos, $16,7 \%$ (9) são graduados, $35,2 \%$ (19) são mestres, $31,5 \%$ (17) são especialistas, $5,6 \%$ (3) são doutores e $5,6 \%$ (3) possuem pós-doutorado.

\subsection{Procedimento de coleta}

A pesquisa foi divulgada pela Internet no ambiente Google Docs e durou um mês (novembro de 2013). Nesse período foram enviados dois lembretes em datas diferentes convidando para responder a pesquisa, antes de encerrar a coleta de dados (CRESWELL, 2007).

\subsection{Método de análise}

Para a realização da análise fatorial foram checados os pressupostos de normalidade, linearidade e multicolinearidade, análise exploratória dos dados, inspeção de outliers e verificação da singularidade (NEIVA; ABBAD; TRÓCCOLI, 2007). Seguiu-se a verificação da fatorabilidade da matriz de correlações para cada amostra, verificação do determinante da matriz e cálculo do índice de adequação da amostra de Kaiser-Meyer-Olkin (teste de KMO). Para avaliar a estabilidade, a consistência das escalas e a validade dos construtos das variáveis, foi usada a análise exploratória dos fatores e análise de confiabilidade (TABACHNICK; FIDELL, 2001). Os escores dos respondentes para cada construto foram obtidos pela soma de todos os itens nas variáveis do construto (SEK et al., 2010). 


\section{RESULTADOS}

\subsection{Validação do instrumento}

Antes da coleta de dados final, foram analisadas as medidas das escalas para determinar a confiabilidade e validade discriminante. A análise da confiabilidade dos modelos de medição foi determinada por meio da consistência interna com base no Alfa de Cronbach (TABACHNICK; FIDELL, 2001). Conforme apresentado na Tabela 1, o coeficiente Alfa dos construtos variou de 0,82 a 0,92. Esses índices de confiabilidade são considerados bons (PASQUALI, 2012).

Tabela 1 - Estatística descritiva e análise de confiabilidade

\begin{tabular}{l|c|c|c}
\hline \multicolumn{1}{c|}{ Construtos } & $\begin{array}{c}\text { Média } \\
(\overline{\boldsymbol{x}})\end{array}$ & $\begin{array}{c}\text { Desvio Padrão } \\
(\boldsymbol{\sigma})\end{array}$ & $\begin{array}{c}\text { Alfa de Cronbach } \\
(\boldsymbol{\alpha})\end{array}$ \\
\hline Facilidade de Uso Percebida (FUP) & 3,91 & 0,89 & 0,82 \\
\hline Utilidade Percebida (UP) & 4,06 & 0,89 & 0,91 \\
\hline Atitude para Uso (AU) & 4,39 & 0,70 & 0,86 \\
\hline Intenção Comportamental (IC) & 4,41 & 0,74 & 0,92 \\
\hline
\end{tabular}

Fonte: Dados da pesquisa, 2013

A análise exploratória dos fatores foi realizada para determinar a validade discriminante das escalas. Um total de 21 itens foram analisados para avaliar as 5 escalas, sendo 6 para Facilidade de Uso Percebida; 6 para Utilidade Percebida; 4 para Atitude em Relação ao Uso; 3 para Intenção Comportamental e 2 de múltipla escolha para uso real.

A variável Facilidade de Uso Percebida, apesar de ser a média mais baixa $(\bar{x}=3,91, \sigma=0,89)$, indica que a computação em nuvem parece ser fácil de usar. Também houve concordância em relação a Utilidade Percebida, Atitude para Uso e Intenção Comportamental. Para avaliar o Uso Real foi considerada a frequência de uso das ferramentas: 96,4\% utilizam algum tipo de recurso de computação em nuvem (e-mail, armazenamento, agenda, grupos, contatos, bate papo e web conferência). Os demais (duas pessoas) não utilizam qualquer uma dessas ferramentas. Quanto à frequência (questão de múltipla escolha com possibilidade de mais de uma escolha) de uso dessas ferramentas, 96,2\% usam e-mail, $75,5 \%$ contatos, $73,6 \%$ agenda, $64,2 \%$ armazenamento e $58,5 \%$ conversas instantâneas do tipo bate papo.

\subsection{Análise Fatorial}

Para verificar a fatorabiliade da matriz de correlações foram realizados os seguintes procedimentos: inspeção da matriz de correlações; verificação do determinante da matriz e cálculo do índice de adequação da amostra de Kaiser-Meyer-Olkin. A estatística do teste de $\mathrm{KMO}$ varia entre 0 e 1 . Considera-se um valor superior a 0,50 como um ponto de corte para proceder com a análise fatorial (HAIR et al., 2006). Por outro lado, Tabachnick e Fidel (2001, p. 589), consideram que "valores de 0,60 e acima são necessários para uma boa análise fatorial". Conforme mostrado na Tabela 2, todas as variáveis alcançaram índices satisfatórios para esse critério. 
Helga Cristina Hedler et al.

Tabela 2 - Resultado dos testes de KMO e de Bartlett dos construtos

\begin{tabular}{l|l|c|c|c|c}
\hline \multicolumn{2}{c|}{ Testes } & FUP & UP & AU & IC \\
\hline \multicolumn{2}{l|}{ Kaiser-Meyer-Olkin (KMO) } & 0,77 & 0,75 & 0,77 & 0,73 \\
\hline \multirow{2}{*}{$\begin{array}{l}\text { Teste de } \\
\text { esfericidade de } \\
\text { Bartlett }\end{array}$} & $\begin{array}{l}\text { Qui- } \\
\text { quadrado }\end{array}$ & 106,72 & 201,48 & 103,03 & 97,68 \\
\cline { 2 - 5 } & df & 15 & 15 & 6 & 3 \\
\cline { 2 - 5 } & Significância & 0,00 & 0,00 & 0,00 & 0,00 \\
\hline
\end{tabular}

Fonte: Dados da pesquisa, 2013

Facilidade de Uso Percebida (FUP) apresentou estrutura unifatorial, cuja escala corresponde a $46 \%$ de variância explicada pelo instrumento. $\mathrm{O} \mathrm{KMO}$ foi 0,77 , ou seja, a escala apresenta uma variância explicada baixa. Isto poderia ser ocasionado pela necessidade de um número maior de itens, que poderiam melhorar a explicação do construto e compor a medida do instrumento. As cargas fatoriais, apresentadas na Tabela 3 variaram entre 0,544 a 0,821.

Tabela 3 - Resultado Final da Análise Fatorial (Método de extração: Fatoração dos Eixos Principais - PAF)

\begin{tabular}{cc}
\hline Escalas & Carga fatorial \\
\hline FUP $_{1}$ & 0,681 \\
FUP $_{2}$ & 0,745 \\
FUP $_{3}$ & 0,683 \\
FUP $_{4}$ & 0,544 \\
FUP $_{5}$ & 0,554 \\
FUP $_{6}$ & 0,821 \\
\hline $\mathrm{AU}_{1}$ & 0,730 \\
$\mathrm{AU}_{2}$ & 0,723 \\
$\mathrm{AU}_{3}$ & 0,841 \\
$\mathrm{AU}_{4}$ & 0,858 \\
\hline
\end{tabular}

\begin{tabular}{cc}
\hline Escalas & Carga fatorial \\
\hline$U P_{1}$ & 0,694 \\
$U P_{2}$ & 0,928 \\
$U P_{3}$ & 0,848 \\
$U P_{4}$ & 0,777 \\
$U P_{5}$ & 0,777 \\
$U P_{6}$ & 0,727 \\
\hline$I C_{1}$ & 0,923 \\
$I C_{2}$ & 0,896 \\
$I C_{3}$ & 0,789 \\
\hline
\end{tabular}

Fonte: Dados da pesquisa, 2013

Utilidade Percebida (UP) apresentou KMO de 0,75, estrutura unifatorial e a escala corresponde a $63 \%$ da variância explicada. As cargas fatoriais variaram entre 0,69 e 0,93. Atitude para Uso (AU) apresentou índice $\mathrm{KMO}$ de 0,77 , a variância explicada pelo escala foi de $62,5 \%$ e as cargas fatoriais variaram entre 0,72 e 0,86 .

Intenção Comportamental (IC) apresentou índice de KMO de 0,73, variância explicada pelo instrumento de $76 \%$, cargas fatoriais variando entre 0,79 e 0,92 . As cargas fatoriais e a variância explicada indicam que os instrumentos possuem indícios de validade.

Cogitando-se a possibilidade que o tamanho da amostra tenha afetado o resultado da análise fatorial, salienta-se que se um fator é representado por um bom número de itens (quatro ou mais) e se esses itens são fortemente explicados pelo fator, ou seja, possuem cargas fatoriais elevadas $(>0,60)$, o número de respondentes tende a ser menos importante na obtenção de uma boa estrutura fatorial (DAMÁsIO, 2012). A escala FUP possui dois itens com cargas baixas ( $\mathrm{FUP}_{4}$ e $\mathrm{FUP}_{5}$ ), o que pode indicar necessidade de reformulação ou substituição desses itens nessa escala.

\section{DISCUSSÃO E CONCLUSÃO}

Quanto à avaliação da aceitação da computação em nuvem pelos usuários na organização, a análise descritiva indica uma alta incidência e frequência de uso. Os escores acima de 4 indicam concordância em relação à Utilidade Percebida $(\bar{x}=4,06, \sigma=0,89)$, à Atitude para Uso $(\bar{x}=4,39, \sigma=0,70)$ e à Intenção Comportamental $(\bar{x}=4,41, \sigma=0,74)$. A 
Facilidade de Uso da computação em nuvem não teve um escore tão elevado quanto os demais construtos do modelo $(3,91, \sigma=0,89)$, podendo estar associada a diversos fatores, como uma não compreensão global do que vem a ser utilizar aplicativos em nuvem.

Em relação ao modelo avaliado, as quatro escalas avaliadas - Intenção Comportamental $(\alpha=0,92)$, Utilidade Percebida $(\alpha=0,91)$, Atitude para Uso $(0,86)$ e Facilidade de Uso Percebida $(\alpha=0,82)$ - obtiveram bons níveis de confiabilidade. No entanto, a escala FUP possui dois itens com cargas fatoriais baixas $\left(\mathrm{FUP}_{4}=0,544\right.$ e $\left.\mathrm{FUP}_{5}=0,554\right)$. Por este motivo, entende-se que é preciso reformular ou criar mais itens para avaliar esse fator. A literatura mostra que o modelo tem sido amplamente testado e suportado nessas aplicações. Assim, um número de itens reduzido pode ser adequado. No entanto, pode haver, no contexto da computação em nuvem, maior necessidade de itens para melhor entendimento da tecnologia avaliada, dada a sua complexidade conceitual para os usuários finais.

A proposição de organização da matriz em três fatores corrobora a proposta evolutiva do TAM dada por Davis, Bagozzi e Warshaw (1989), quando sinalizam que a relação de atitude com intenção comportamental, fundamental para a TRA e para os modelos relacionados, poderia ser direta, ou seja, a variável Utilidade Percebida poderia ser preditora da intenção comportamental.

Cabe destacar Venkatesh e Davis (2000) que, no trabalho que originou o TAM2, propõem a exclusão do construto de Atitude para Uso, numa tentativa de tentar explicar melhor a intenção comportamental, mantendo o corpo principal do modelo com somente três fatores. Essa pesquisa não encontrou variância na variável Atitude para Uso, reforçando o posicionamento de Venkatesh et al (2003), que mantiveram o modelo sem essa variável.

Vários motivos podem ser considerados para entender que a medida Atitude para o Uso não obteve índice razoável para validação do modelo TAM proposto por Sek et al (2010) e utilizado besta pesquisa. Acredita-se que o tipo de tecnologia para o qual se aplicou o modelo pode ser um dos motivos. Por exemplo, enquanto Davis (1989) e Sek et al (2010) avaliaram dispositivos ou uma única ferramenta, esta pesquisa avaliou uma tecnologia mais complexa, que envolve hardware e software de forma conjunta, de maneira supostamente menos tangível para o participante, ou menos entendida enquanto conceito complexo. Se computadores ou smartphones são objetos físicos palpáveis, a computação em nuvem é um composto de vários possíveis objetos e uma gama de aplicações e conceitos que se referem à tecnologia.

Quanto às crenças, segundo Davis (1986), estas se referem à Utilidade Percebida e Facilidade de Uso Percebida, sendo que a facilidade influencia a utilidade. A Utilidade Percebida reforça a ideia de que os usuários acreditam que computação em nuvem é útil para o desempenho de suas atividades, haja vista que, para esse fator, $\bar{x}=4,06(\sigma=0,89)$. As crenças dos usuários são favoráveis em relação à Facilidade de Uso da computação em nuvem e que a computação em nuvem é fácil de utilizar $(\bar{x}=3,91, \sigma=0,89)$.

O uso da computação em nuvem, diferente de outras tecnologias, não requer uma significativa alteração de procedimentos ou de aquisição de novas competências e habilidades por parte de seus usuários. Isso pode ser favorável à aceitação e uso dessa tecnologia pelo fato de que, muitas vezes, o próprio usuário não percebe diretamente alterações na sua rotina de trabalho.

Nota-se que Davis, Bagozzi e Warshaw (1989) não incluíram no TAM a norma subjetiva como determinante da Intenção Comportamental. No entanto, é possível que os participantes da pesquisa tenham usado o sistema meramente para cumprir as ordens da chefia (normas subjetivas) e não devido às suas crenças sobre o uso da computação em nuvem.

Diante dos resultados encontrados, é relevante retomar as recomendações de Benbasat e Barki (2007) ao proporem que é tempo de pesquisadores interessados no tema 
proporem novos modelos. Também cabe destacar que Chuttur (2009) concorda com esta visão ao considerar que, pelo TAM ter sido tão experimentado, é hora de avaliar novos modelos que explorem suas forças e minimizem suas fraquezas.

Como limitação desta pesquisa, frisamos que não foi possível a adaptação do modelo de aplicação do TAM baseado no instrumento de pesquisa de Sek et al (2010), uma vez que este não se comportou adequadamente ao se avaliar a aceitação de computação em nuvem e que não foi possível testar as relações preditas no modelo. Neste sentido, tende-se a reforçar a necessidade de inclusão de novos fatores, com a criação de novos itens que enfatizem o processo comportamental na adoção de computação em nuvem.

Como proposição de uma agenda para estudos futuros, recomenda-se que sejam revisadas as pesquisas de Venkatesh e Davis (2000), que propuseram uma nova representação do modelo TAM, denominado TAM2, que mantinha a essência de Davis (1986). Apesar do modelo TAM2 não ter sido utilizado nesta pesquisa, ele é um bom exemplo de novas possibilidades de composição fatorial a partir do corpo essencial do TAM e de outras teorias para investigação de fenômenos relacionados à aceitação de tecnologia, assim como o modelo UTAUT (VENKATESH et al, 2003), o modelo de pesquisa de Amoako-Gyampah e Salam (2003) ou o modelo de Wixom e Tood (2005), que poderiam auxiliar a composição de novos modelos de pesquisa.

Entre novas variáveis que poderiam ser testadas para a adaptação de um instrumento de pesquisa direcionado para a temática da aceitação de computação em nuvem com base no TAM poderiam estar fatores relacionados ao indivíduo (comprometimento, resistência à tecnologia, capacitação) e à própria organização, como cultura, mudança, expectativa de desempenho, condições facilitadoras, incentivos, tipo de personalidade - se inovadora ou não, e maneira de implementação da tecnologia. Sugere-se ainda que em estudos futuros que envolvem a aceitação e uso da computação em nuvem ou similar, seja considerada a influência das normas subjetivas.

Além da proposição de novos itens para o instrumento de pesquisa para verificar a perspectiva comportamental da adoção de computação em nuvem, uma agenda de estudos futuros pode envolver também a criação ou adaptação de novos modelos, da realização de novas aplicações de questionários, em diferentes contextos e de novos métodos que visem contribuir para que as organizações possam melhor lidar com a computação em nuvem. Como afirmam Khajeh-Hosseini, Sommerville e Sriram (2012a), é importante a atenção aos desafios de ordem técnica, como segurança de informação, protocolos de transferência de dados, desempenho de sistemas, escalabilidade. Também é necessário desenvolver uma perspectiva humanística, haja vista que a computação em nuvem não é simplesmente uma melhoria tecnológica dos centros de dados, mas uma mudança fundamental na forma como a Tl é ofertada e usada.

\section{REFERÊNCIAS}

ALHARBI, S. T. Users' Acceptance of Cloud Computing in Saudi Arabia: an extension of technology acceptance model. International Journal of Cloud Applications and Computing, v. 2, n. 2 p. 1-11, 2012.

AMOAKO-GYAMPAH, K.; SALAM, A. F. An extension of the technology acceptance model in an ERP implementation environment. Information \& Management, v. 4, n. 3, p. 11-23, 2003.

ARMBRUST, M. et al. Above the Clouds: a Berkeley view of cloud computing. Technical Report. University of California at Berkeley. 2009. Disponivel em: https://www2.eecs.berkeley.edu/Pubs/TechRpts/2009/EECS-2009-28.pdf 
ARMENISE, R. et al. The design of a Decision Support Tool for Delivery and Deployment Models. In: IEEE SYMPOSIUM ON NETWORK CLOUD COMPUTING AND APPLICATIONS, 3., 2014. Proceedings ... p. 91-94, 2014.

AZEEMI, I.K.; LEWIS, M.; TRYFONAS, T. Migrating To The Cloud: Lessons And Limitations Of "Traditional" IS Success Models. Procedia Computer Science, v. 16, p. 737-746, 2013.

BENBASAT, I.; BARKI, H. "Quo vadis TAM?". Journal of the Association for Information Systems, v. 8, n. 4, 2007.

BETIOL, M. I. S.; TONELLI, M. J. Absenteísmo e Comprometimento: algumas reflexões a partir de um estudo de caso sob a ótica da Psicodinâmica do Trabalho. In: ENCONTRO NACIONAL DE ENGENHARIA DE PRODUÇÃO - ENEGEP, 2001, Salvador. Anais... Salvador: ABEPRO, 2001. Disponível em: http://www.abepro.org.br/biblioteca/ENEGEP2001 TR44 0657.pdf

BUYYA, R. et al. Cloud computing and emerging IT platforms: vision, hype, and reality for delivering computing as the 5th utility. Vienna: Institute of Information Systems, University of Technology, 2012.

CARR, N. It doesn't Matter. Harvard Business Review, v. 81, n. 5, p. 41-49, 2003.

CHAVES, S.; SOUZA, C. A. de. Benefícios potenciais associados à computação em nuvem: um estudo delphi envolvendo profissionais e acadêmicos brasileiros. In: SEMINÁRIOS EM ADMINISTRAÇÃO - SEMEAD, 15., 2012, São Paulo. Anais.... São Paulo, 2012. Disponível em: http://sistema.semead.com.br/15semead/resultado/trabalhosPDF/919.pdf

CHUTTUR, M. Y. Overview of the Technology Acceptance Model: origins, developments and future directions. Indiana University: USA, Sprouts: Working Papers on Information Systems, 2009.

CRESWELL, J. W. Projeto de pesquisa: métodos qualitativo, quantitativo e misto. 2. ed. Porto Alegre: Artmed, 2007.

DAMÁSIO, B. F. Uso da análise fatorial exploratória em psicologia. Avaliação Psicológica, v. 11, n. 2, p. 213-228, 2012. Disponível em: http://pepsic.bvsalud.org/pdf/avp/v11n2/v11n2a07.pdf

DAVIS, F. D. A technology acceptance model for empirically testing new end-user information systems: theory and results. Tese (Doutorado)- Sloan School of Management, Massachusetts Institute of Technology, 1986. Disponível em: http://dspace.mit.edu/handle/1721.1/15192

DAVIS, F. D. Perceived usefulness, perceived ease of use, and user acceptance of computer technology. MIS Quarterly, v. 13, n. 3, p. 319-340, 1989.

DAVIS, F. D.; BAGOZZI, R. P.; WARSHAW, P. R. User acceptance of computer technology: a comparison of two theoretical models. Management Science, v. 35, n. 8, p. 982-1003, 1989.

DeLONE, W. H.; McLEAN, E. R. Information systems success: the quest of dependent variable. Information Systems Research, v. 3, n. 1, p. 60-95, 1992.

ETRO, F. The Economic Impact of Cloud Computing on Business Creation, Employment and Output in Europe: an application of the endogenous market structures approach to a GPT innovation. Review of Business and Economic, v. 3, p. 179-209, 2009.

FISHBEIN, M.; AJZEN, I. Belief, attitude, intention, and behavior: an introduction to theory and research. Boston (MA): Addison-Wesley, 1975.

GONZENBACH, I.; RUSS, C.; BROCKE, J. von. Make or Buy? Factors that Impact the Adoption of Cloud Computing on the Content Level. In: BROCKE, J. von; SIMONS, A. (eds). Enterprise 
Content Management in Information Systems Research. Progress in IS. Berlin, Heidelberg: Springer Berlin Heidelberg, p. 145-161, 2014.

HAIR, J.F. et al. Multivariate Data Analysis. 6. ed. Upper Saddle River, NJ: Pearson Prentice Hall, 2006.

HATCH, M. J. The Dynamics of Organizational Culture. The Academy of Management Review, v. 18, n. 4, p. 657-693, 1993. Disponível em: http://www.jstor.org/stable/258594.

JOHNSON, B.; QU, Y. A Holistic Model for Making Cloud Migration Decision: A Consideration of Security, Architecture and Business Economics. In: IEEE INTERNATIONAL SYMPOSIUM ON PARALLEL AND DISTRIBUTED PROCESSING WITH APPLICATIONS, 10., 2012. Proceedings..., p. 435-441, 2012.

KHAJEH-HOSSEINI, A. et al. Decision Support Tools for Cloud Migration in the Enterprise. In: IEEE INTERNATIONAL CONFERENCE ON CLOUD COMPUTING, 4., 2011. Proceedings ..., p. 541$548,2011$.

KHAJEH-HOSSEINI, A.; SOMMERVILLE, I; SRIRAM, I. Research Challenges for Enterprise Cloud Computing. [Computer-Communication Networks]: Distributed Systems - Cloud computing, 2012. Disponível em: http://arxiv.org/ftp/arxiv/papers/1001/1001.3257.pdf.

KHAJEH-HOSSEINI, A. et al.The Cloud Adoption Toolkit: supporting cloud adoption decisions in the enterprise. Software: Practice and Experience, v. 42, n. 4, p. 447-465, 2012a.

MANYIKA, J. et al. Disruptive technologies: Advances that will transform life, business, and the global economy. McKinsey Global Institute, 2013.

MARSTON, S. et al. Cloud computing - the business perspective. Decision Support Systems, v. 51, p. 176-189, 2011.

MARTENS, B.; TEUTEBERG, F. Decision-making in cloud computing environments: A cost and risk based approach. Information Systems Frontiers, v. 14, n. 4, p. 871-893, 2011.

McGEE, J.; PRUZAK, L. Gerenciamento estratégico da informação. Rio de Janeiro: Campus, 1997.

MELL, P.; GRANCE, T. The NIST Definition of Cloud Computing. Computer Security Division, Information Technology Laboratory, National Institute of Standards and Technology, United States Department of Commerce. Gaithersburg, MD 20899-8930: National Institute of Standards and Technology, 2011.

MILLER, M. Cloud Computing: pros and cons for end users. Pearson - QUE. 2009. Disponível em: http://www.quepublishing.com/articles/article.aspx?p=1324280.

MORRIS, F. Infographic: SMB Cloud Adoption Trends in 2014. PCWorld, 7 de Outubro de 2014. Disponível em: http://www.pcworld.com/article/2685792/infographic-smb-cloud-adoptiontrends-in-2014.html.

NEIVA, E. R.; ABBAD, G.; TRÓCCOLI, B. T. Roteiro para análise fatorial de dados. Manuscrito não publicado, Instituto de Psicologia, Universidade de Brasília, DF, 2007.

O'REILLY, T. What is web 2.0?: design patterns and business models for the next generation of software. 2012. Disponível em: http://oreilly.com/web2/archive/what-is-web-20.html.

PASQUALI, L. Análise Fatorial para Pesquisadores. Brasília: LabPAM, 2012.

PIRES, F. R. Planejamento para gestão da tecnologia da informação para uso estratégico na pequena empresa. In: CONGRESSO VIRTUAL BRASILEIRO DE ADMINISTRAÇÃO - CONVIBRA,

Perspectivas em Gestão \& Conhecimento, João Pessoa, v. 6, n. 2, p. 188-207, jul./dez. 2016. 
Anais ... 2007. Disponível em: http://www.convibra.com.br/2007/congresso/artigos/308.pdf.

PORRAS, J. I.; ROBERTSON, P. J. Organizational development: theory, practice, and research. In: Dunnette, M. D. \& Hough, L. M. (Org.). Handbook of Industrial and organizational Psychology, p. 719-822, Palo Alto: Consulting Psychologists Press, 1992.

PORTER, M. E. Estratégia competitiva: técnicas para a análise da indústria e da concorrência. Rio de Janeiro: Campus, 1986.

RAJ, P.; PERIASAMY, M. The Cloud Challenges for Enterprise Architects. In: Mahmood, Z.; Hill, R. (Eds). Cloud Computing for Enterprise Architectures. Londres: Springer-Verlag, 2011. p. 187-206, 2011.

RAPPA, M. Business Model on the Web. Managing the digital enterprise. 2004. Disponível em: http://www.startupjunkies.org/business models.pdf.

ROGERS, E. M. Diffusion of Innovations. 4 ed., New York: The Free Press, 1995.

SAGA, V. L.; ZMUD, R. W. The Nature and Determinants of IT Acceptance, Routinization, and Infusion. In: IFIP TC8 WORKING CONFERENCE ON DIFFUSION, TRANSFER AND IMPLEMENTATION OF INFORMATION TECHNOLOGY. Proceedings ... New York: Elsevier Science, 1993. p. 67-86.

SANTOS JUNIOR, S.; FREITAS, H.; LUCIANO, E. M. Dificuldades para o uso da tecnologia da informação. RAE-eletrônica, v. 4, n. 2, 2005. Disponível em: $<$ http://www.scielo.br/pdf/raeel/v4n2/v4n2a05.pdf>

SEK, Y.-W. et al. Prediction of user acceptance and adoption of smartphone for learning with technology acceptance model. Journal of Applied Sciences, v. 5, p. 2395-2402, 2010.

SHAPIRO, C.; VARIAN, H. R. Information rules, a strategic guide to the network economy. Boston: Harvard Business School Press, 1999.

TABACHNICK, B. G.; FIDELL, L. S. Using Multivariate Statistics. 4. ed. Boston: Allyn and Bacon, 2001.

TAURION, C. Computação em nuvem: transformando o mundo da tecnologia da informação. Rio de Janeiro: Brasport, 2009.

TORRES, I. V. Sucesso da tecnologia da informação em pequenas e médias empresas: proposição conceitual e a percepção de gestores. Dissertação (Mestrado em Administração) Universidade Federal da Paraíba, João Pessoa, 2009.

VELTE, A.T.; VELTE, T.J.; ELSENPETER, R. Computação em nuvem: uma abordagem prática. Rio de Janeiro: Alta Books, 2012.

VENKATESH, V.; DAVIS, F. D. A theoretical extension of the technology acceptance model: Four longitudinal field studies. Management Science, v. 46, n. 2, p. 186-204, 2000.

VENKATESH, V. et al. User acceptance of information technology: towards a unified view. MIS Quarterly, v. 3, p. 425-478.

WIXOM, B. H.; TODD, P. A. A theoretical integration of user satisfaction and technology acceptance. Information Systems Research, v. 16, n. 1, p. 85-102, 2005.

YAM, C.-Y. et al. Migration to Cloud as Real Option: Investment Decision under Uncertainty. In: IEEE INTERNATIONAL CONFERENCE ON TRUST, SECURITY AND PRIVACY IN COMPUTING AND COMMUNICATIONS - TRUSTCOM, 10. Proceedings ..., p. 940-949, 2011. 
ZITTRAIN, J. Saving the Internet. Harvard Business Review, v. 4, p. 49-59, 2007. 